\title{
Direct Ocean Surface Velocity Measurement for Chinese Gaofen-3 SAR Satellite
}

\author{
Lei Liu ${ }^{\# 1}$, Mihai Datcu ${ }^{* 2}$, Qingjun Zhang ${ }^{\# 3}$, Gottfried Schwarz ${ }^{* 4}$, Jie Liu ${ }^{\# 5}$, Yadong Liu ${ }^{\# 6}$ \\ \# Institute of Spacecraft System Engineering, China Academy of Space Technology, China \\ ${ }^{*}$ Remote Sensing Technology Institute, German Aerospace Centre (DLR), Germany \\ \{1liulei211, ${ }^{3}$ ztzhangqj\}@163.com, ${ }^{2}$ Mihai.Datcu@dlr.de, ${ }^{4}$ Gottfried.Schwarz@web.de, ${ }^{5}$ liujie@cast.cn, \\ ${ }^{6}$ liuyadong_1983@126.com
}

\begin{abstract}
Spaceborne Synthetic Aperture Radar (SAR) is one important way to obtain the information of ocean surface velocity. The Doppler Centroid Anomaly (DCA) estimation, which obtain the radical velocity based on the difference between the measured Doppler centroid and the predicted Doppler centroid, have be used to most onboard spaceborne SAR. GaoFen-3 (GF-3), the first full-polarimetric SAR satellite of China. This paper shows the experiments of direct ocean surface velocity measurement for GF-3 SAR Satellite. Comparing the results to the actual ocean current, the proposed method of ocean surface velocity measurement for GF-3 SAR is validated. Thus, GF-3 has the similar ability to retrieve the radical ocean surface velocity as Sentinel-1 and Radarsat-2.
\end{abstract}

Keywords - ocean surface velocity, SAR, Doppler centroid, Gaofen-3.

\section{INTRODUCTION}

Spaceborne Synthetic Aperture Radar (SAR) is one important way to obtain the information of ocean surface feature, in [1], e.g. ocean winds, waves, surface current, internal wave, oil film, mesoscale eddies, fronts, ships and so on. The ocean currents reflect the movement of ocean water, which are created by wind, water temperature, salt content and the gravity of the moon. Thus, routine monitoring the ocean current is of great interest both scientific and coastal communities. In particular, current shear and convergence zones can modulate the distribution of ocean waves, resulting in wave steepening and enhanced ocean surface roughness changes.

During the past decades, there are two methods for measuring ocean surface velocity with SAR which have been discovered and validated in [2]. One is Along Track Interferometry (ATI), which requires two receiver antennas spaced along the satellite track to obtain the echo of the ocean surface at the same time. SRTM and TerraSAR have carried out the onboard ATI experiments to measure the radical current, which is present in [3] and [4]. Based on the Aperture Switching, the along baseline of TerraSAR is $1.3 \mathrm{~m}$ to measure the radical ocean velocity. Simulations and experiments show TerraSAR can measure the surface current with $0.1 \mathrm{~m} / \mathrm{s}$ accuracy in $1 \mathrm{~km}$ spatial resolution.

The other method is single-antenna Doppler shift method called Doppler Centroid Anomaly (DCA) estimation in [2] and [6], which obtains the radical velocity based on the difference between the measured Doppler centroid and the predicted Doppler centroid. DCA has be used to most onboard spaceborne
SAR, include ERS-1, ERS-2, Envisat, Radarsat-1, Sentinel-1A (launched in 2016) and Sentinel-1B (launched in 2018). In particular, Sentinel-1 have open the high-precision products of ocean surface wind and current, called OCN in [7], which promotes DCA method wide and routine application.

Variant ocean current can change the velocity distribution of ocean surface waves, resulting in the change of Doppler spectrum. The direct Doppler measurement of ocean surface current is proposed in [8] and put forward theoretically and experimentally in spaceborne Radarsat-1 SAR in [6]. The Doppler Centroid is proved to be consist of the main contribution of wind-driven wave and ocean surface current that is shown in [2]. According to the difference between the estimated Doppler centroid and the predicted one, the global ocean radical velocity can be retrieved using several years' Envisat images.

DopRIM (Doppler Radar Image Model) in [9] has been proposed to high precision current inversion, in which the Doppler shift results from the combined action of near surface wind on shorter waves, longer wave motion, wave breaking and surface current. ESA began to promote the application of DCA in current inversion in ENVISAT satellite since 2006. DopRIM is successfully used to process WSM (Wide Swath Medium) images of ASAR. The work in [5] have found that the RSM of Doppler shift is $4.7 \mathrm{~Hz}$ and $3.9 \mathrm{~Hz}$ for VV and $\mathrm{HH}$, respectively. The error of DopRIM in ocean surface current inversion is $0.23 \mathrm{~m} / \mathrm{s}$ and $0.19 \mathrm{~m} / \mathrm{s}$ with the incidence angle of $35^{\circ}$ for $\mathrm{VV}$ and $\mathrm{HH}$, respectively.

A high-precision Doppler centroid anomaly estimator in [10] was developed and implemented as part of the Sentinel-1 Level 2 ocean processor. This gives an accuracy in the corresponding radical velocity projected on the ocean surface of less than 0.2 $\mathrm{m} / \mathrm{s}$ on a spatial resolution down to $2 \times 2 \mathrm{~km}^{2}$ and proves Doppler standard deviation between $2.49 \mathrm{~Hz} \sim 2.89 \mathrm{~Hz}$ over homogenous ocean areas.

GaoFen-3 (GF-3), the first full-polarimetric SAR satellite of China with a resolution up to $1 \mathrm{~m}$, had been successfully launched in August 2016 and, after 5 months of in-orbit calibration, it had been officially delivered to the users in January 2017 that is shown in [11]. The SAR images of GF-3 have been applied in wind inversion, oil spill detection, internal waves observation over more than three years. This paper shows the experiments of direct ocean surface velocity measurement for GF-3 SAR Satellite. The main algorithms and 
procedures are described in Section II. Section III shows the results of ocean surface velocity based on GF-3 single-look complex data in fine-strip model. Finally, the conclusion and outlook of future work are present in section IV.

\section{AlgORITHM BASICS}

\section{A. Doppler centroid anomaly model for current measurement}

The predicted geometrical Doppler shift $f_{D p}$ is given by the relative velocity of the satellite and the rotating Earth for loworbit SAR satellite [5].

If the satellite orbit and attitude remain stable, the measured Doppler shift $f_{D c}$ can be estimated from the SAR raw data or processed data. The anormal Doppler frequency shift $f_{D a}$ is

$$
f_{D a}=f_{D c}-f_{D p}
$$

Taking into account the errors $f_{\text {Derr }}$ in the Doppler centroid estimation, the geographic Doppler shift $f_{g}$ caused by ocean motion shows as followed.

$$
f_{g}=f_{D a}-f_{\text {Derr }}
$$

If the observation scene is stationary (such as land), $f_{g}=0$. If the observation scene is moving (such as the ocean), $f_{g}$ contains the motion information of the ocean scatterer. The radical velocity of ocean surface current could be estimated by $f_{g}$ according to DopRIM model.

The DopRIM model [9] establishes a two-scale ocean scattering and Doppler spectral model, which considers the sea backscattering coefficients as the ones modulated by the long waves and the surface current. Meanwhile, the sea surface scatterer (Bragg waves) is moving with long waves and current. The radical velocity of ocean surface $V_{D}$ is

$$
V_{D}=-\frac{\pi f_{g}}{k_{r}} \sin \theta=\overline{c_{f}}+u_{c}+c_{f}^{T H}
$$

Where $k_{r}$ is the radar wave number, $\theta$ is the incidence angle, $\overline{c_{f}}$ is the average phase velocity of the wind-driven Bragg waves, $u_{c}$ is the ocean surface velocity, and $c_{f}^{T H}$ is the contribution of hydrodynamic modulation and tilt modulation to the current.

This paper defines that the positive Doppler frequency reflects the current or the waves moving towards the radar. For the descend orbit and the right-sight image geometry, the positive velocity denotes the current moving towards the east.

\section{B. Doppler centroid estimation}

The Average Cross Correlation Coefficient (ACCC) method proposed by [12] is used for Doppler estimation. This method correlates the signals $s(\eta, \tau)$ in the time domain and estimates the centroid of the Doppler spectrum of the signals. The cross-correlation coefficient $c(\eta, \tau)$ is calculated as follows

$$
c(\eta, \tau)=\sum_{\eta} s(\eta, \tau) s^{*}(\eta+\Delta \eta, \tau)
$$

$s(\eta, \tau)$ is the radar signal in time dimension, $\eta$ is the $\mathrm{SAR}$ azimuth time, $\tau$ is the SAR range time; $\Delta \eta=1 / P R F$, PRF is the SAR pulse repetition frequency. The phase of the crosscorrelation coefficient $\phi_{\text {accc }}$ is calculated by the average value of the correlation coefficient in the range direction as follows.

$$
\phi_{\text {accc }}(\eta, \tau)=\arg \left(\sum_{i=1}^{N} c\left(\eta, \tau_{i}\right)\right)
$$

in which $\mathrm{N}$ is the average number of cross correlation coefficients.

The measured Doppler shift $f_{D c}$ based on the SAR signal is

$$
f_{D c}(\eta, \tau)=-\frac{P R F}{2 \pi} \phi_{a c c c}(\eta, \tau)
$$

\section{Segmentation and Quality Assessment}

The data segmentation is used in the processing in order to decrease the amount of calculation. Studies have shown that too high or too low image signal-to-noise ratio (SNR), strong azimuthal radiation gradient, and severe spectral distortion can cause Doppler estimation bias in [13]. Thus, after the SAR data is divided into blocks, the data quality of each block needs to be evaluated. By examining SNR and azimuthal radiation gradient, the data blocks that do not meet quality requirements are removed to ensure estimation accuracy.

SNR is calculated by Harmonic ratio.

$$
H_{\text {ratio }}=20 \log _{10} \frac{a b s\left(S_{1}\right)}{a b s\left(S_{0}\right)}
$$

where $S_{0}$ and $S_{1}$ is the first and second of the Fourier coefficients of the received radar data in the azimuth direction, respectively.

The azimuthal radiation gradient is calculated by the energy gradient of the sub-block in the azimuth direction, when the block data is divided into $4 * 4$ sub-blocks.

\section{PROCESSING RESULTS}

\section{A. Data instruction}

There are more than 12 image models for GF-3 satellite. The SLC data of GF-3 Fine Strip Model II is processed in this paper. In order to evaluate the results, the data that images the northwest coastline of France is chosen to current inversion. The intensity images are shown in Fig.1.

The real-time coastal surface current measured by other methods (updated in the FHOM website https://data.shom.fr/) is used to validate the current measured by GF-3 SAR. Because the radical velocity is most projected to the surface velocity along the range direction, the surface velocity along east-west direction is shown in Fig.2 as the ground truth. 


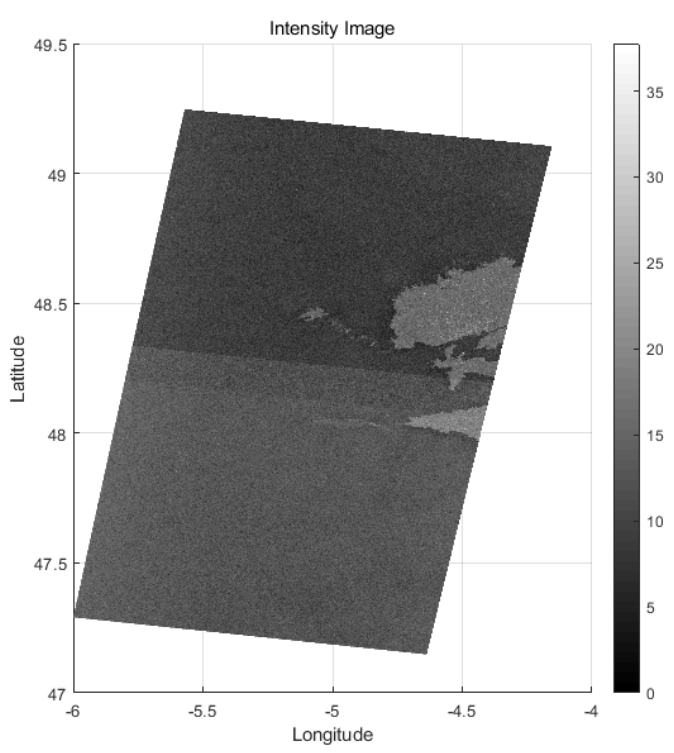

Fig. 1. The intensity of GF-3 images in Fine Strip Model II.

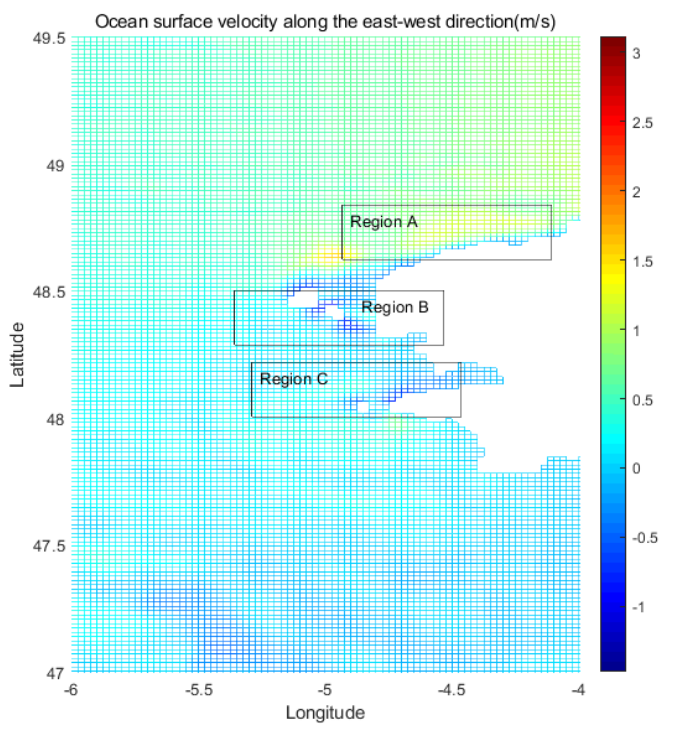

Fig. 2. The surface velocity along east-west direction provided by FHOM.

\section{B. Ocean surface current measured by GF-3}

The measured and geographic Doppler centroid are shown in Fig.3 and Fig.4.

And the ocean surface current is shown in Fig.5. According to the geometry between GF-3 and the observed region, the plus velocity denotes the current with the east direction. The current directions around Region A, B and C are consistent with the actual currents shown in Fig.2.

The ocean surface velocities measured by SAR is slightly higher than the actual ones in Region A, because the phase speed of Bragg waves (likely moving towards to east) has not been subtracted from the ocean surface velocities. The velocities in Region B and C are close to the actual ones, because the wind speed has low magnitude in these regions.

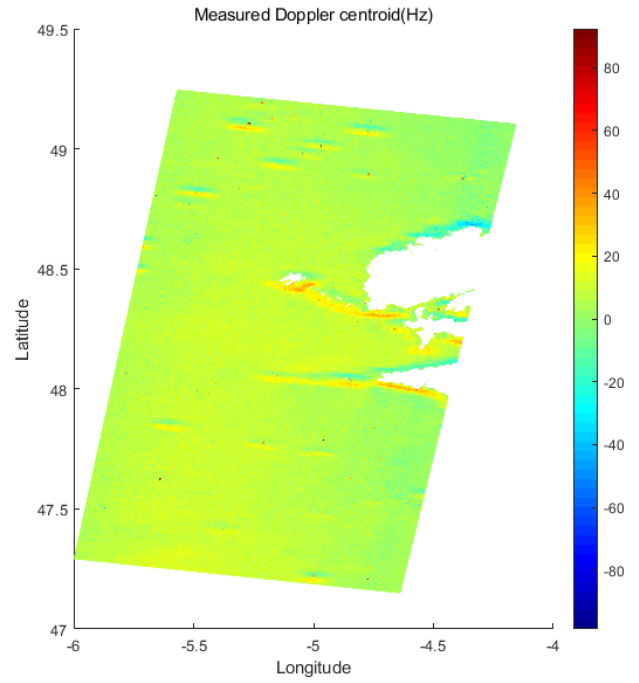

Fig. 3. The measured Doppler centroid based on GF-3 SLC images

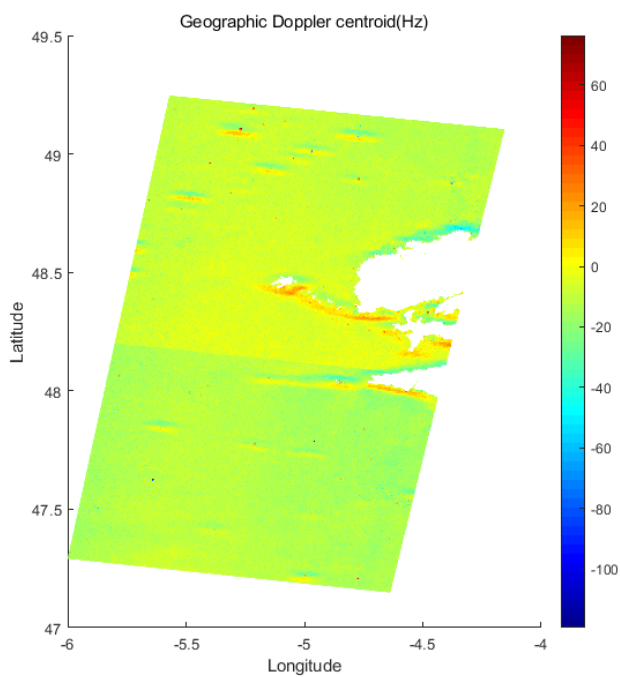

Fig. 4. The geographic Doppler centroid based on GF-3 SLC images

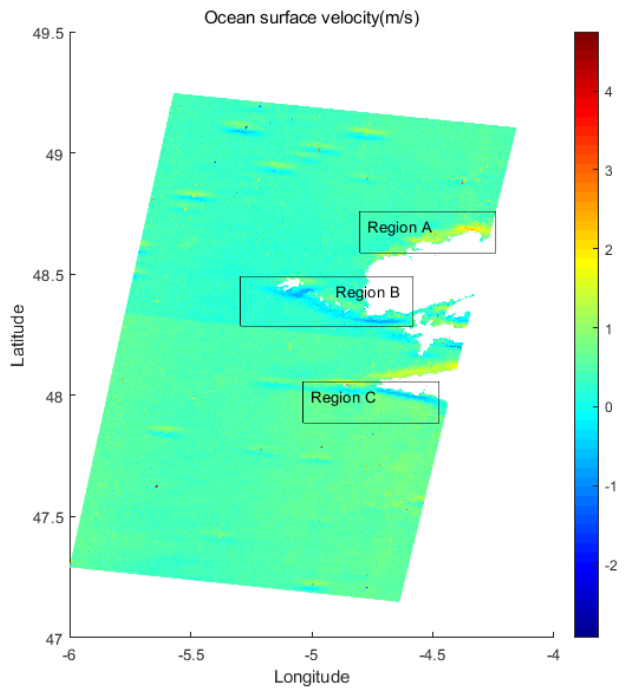

Fig. 5. The ocean surface velocity based on GF-3 SLC images 


\section{CONCLUSION}

The results prove that the proposed method of ocean surface velocity measurement for GF-3 SAR is effective and the GF-3 satellite has similar ability to retrieve the radical ocean surface velocity as compared to Sentinel-1 and Radarsat- 2 .

Future works focus on the comparison GF-3 results to Sentinel-1 OCN product in order to improve the algorithm. Additionally, the subtraction of the speed of wind-driven Bragg wave is also considered in future works.

\section{ACKNOWLEDGMENT}

The authors would like to thank Prof. Gerhard Rigoll and Dr. Yuanyuan Wang from Technical University of Munich whose valuable comments improved this manuscript.

The authors also would like to thank China Centre for Resources Satellite Data and Application for sharing GF-3 data and they thank FHOM for opening the actual current data.

This work was funded by the Chinese Scholarship Council.

\section{REFERENCES}

[1] Christopher R. Jackson and John R. Apel, Synthetic aperture radar: marine user's manual, National Oceanic and Atmospheric Administration, Washington DC, 2004.

[2] Bertrand Chapron, Fabrice Collard, and Fabrice Ardhuin. Direct measurements of ocean surface velocity from space: Interpretation and validation. Journal of Geophysical Research: Oceans, 110(C7), 2005.

[3] Roland Romeiser, Helko Breit, Michael Eineder, Hartmut Runge, Pierre Flament, Karin De Jong, and Jur Vogelzang. Current measurements by sar along-track interferometry froma space shuttle. IEEE Transactions on Geoscience and Remote Sensing, 43(10):2315-2324, 2005.

[4] Roland Romeiser, Steffen Suchandt, Hartmut Runge, UlrichSteinbrecher, and Steffen Grunler. First analysis of terrasar-x along-track insar-derived current fields. IEEE Transactions on Geoscience and Remote Sensing, 48(2):820-829, 2009.

[5] Morten Wergeland Hansen, Fabrice Collard, Knut-Frode Dagestad, Johnny A Johannessen, Pierre Fabry, and Bertrand Chapron. Retrieval of sea surface range velocities from Envisat ASAR Doppler centroid measurements. IEEE Transactions on Geoscience and Remote Sensing, 49(10):3582-3592, 2011.

[6] D. Hutt, J. Stockhausen, J. Osler, and D. Mosher. Capability of radarsat1 for estimation of ocean surface current on the Scotian shelf. IEEE International Geoscience and Remote Sensing Symposium, volume 4, pages 2135-2137. IEEE, 2002.

[7] Harald Johnsen, Vegard Nilsen, Geir Engen, Alex A Mouche, and Fabrice Collard. Ocean doppler anomaly and ocean surface current from sentinel 1 tops mode. In 2016 IEEE International Geoscience and Remote Sensing Symposium (IGARSS), pages 3993-3996. IEEE, 2016

[8] RA Shuchman, CL Rufenach, FI Gonzalez, and A Klooster. The feasibility of measurement of ocean surface currents using synthetic aperture radar. In Thirteenth International Symposium on Remote Sensing of the Environment, Ann Arbor, Michigan, 1979.

[9] Johnny A Johannessen, Bertrand Chapron, Fabrice Collard,Vladimir Kudryavtsev, Alexis Mouche, D Akimov, and K-F Dagestad. Direct ocean surface velocity measurements from space: Improved quantitative interpretation of Envisat ASAR observations. Geophysical Research Letters, 35(22), 2008

[10] Gier Engen and Harald Johnsen. Sentinel-1 doppler and ocean radical velocity (rvl) algorithm definition, Tromsø: Northern Research Institute, 2010.

[11] Qingjun Zhang, Zhenhong Li, Yunkai Deng and Guisheng Liao, Special Issue "First Experiences with Chinese Gaofen-3 SAR Sensor", in Sensor, December 2018.

[12] S. N. Madsen, Estimating the Doppler centroid of SAR data, in IEEE Transactions on Aerospace and Electronic Systems, vol. 25, no. 2, pp. 134-140, March 1989.
[13] Ian G Cumming. A spatially selective approach to doppler estimation for frame-based satellite SAR processing. IEEE Transactions on Geoscience and Remote Sensing, 42(6):1135-1148, 2004 